\title{
ATENCIÓN SELECTIVA VISUAL E INTELIGENCIA: EVIDENCIA DE VALIDACIÓN PARA LA ESCALA DE ATENCIÓN SELECTIVA VISUAL
}

\author{
SELECTIVE VISUAL ATTENTION AND INTELLIGENCE: EVIDENCE OF VALIDITY \\ FOR THE ESCALA DE ATENÇÃO SELETIVA VISUAL
}
ATENÇÃO SELETIVA VISUAL E INTELIGÊNCIA: EVIDÊNCIA DE VALIDADE PARA A ESCALA DE ATENÇÃO SELETIVA VISUAL

Fermino Fernandes Sisto ${ }^{1}$; Nelimar Ribeiro de Castro; Dario Cecillo-Fernandes

Universidad de São Francisco, campus Itatiba, Brasil

(RECiBIDO 12/02/2012; ACEPTADo 16/6/2012)

\begin{abstract}
RESUMEN
O objetivo desta pesquisa foi procurar evidência de validade para a Escala de Atenção Visual. Primeiramente, por diferenças de grupos extremos e, depois, por correlação com o Teste de Raciocínio Inferencial. A pesquisa envolveu 119 universitários com idade média de $22,96(\mathrm{DP}=5,67)$, predominantemente do sexo feminino (79\%). As pontuações da Escala de Atenção Seletiva e Visual (EASE) variaram de quatro a 30 pontos, enquanto as do teste de Raciocínio Inferencial (Rin) estiveram entre zero a 36. Foram comparadas as médias entre os grupos extremos $(t=-2,47 ; g l=63 ; p=0,017)$, cujo resultado indicou que um instrumento discriminou o outro. Ao lado disso, foi constatada uma correlação positiva e significativa $(r=0,24 ; p=0,01)$ entre as pontuações totais dos testes. Portanto, o objetivo do estudo foi alcançado, sendo encontrada uma evidência de validade convergente-discriminante e outra por grupos extremos.
\end{abstract}

Palavras-chaves: teste de atenção, validade, psicometria, avaliação psicológica.

\begin{abstract}
The aim of this research was to look for two kinds of evidence of validity for the Escala de Atenção Seletiva Visual (EASE). Firstly, the means of two contrasted group was analyzed, and then the scale EASE was correlated with the Teste de Raciocínio Inferencial. This research involved 119 students $(\mathrm{M}=22,96$ and $\mathrm{DP}=5,67)$, most of them female $(79 \%)$. The scores of the Escala de Atenção Seletiva e Visual ranged from four to 30 points, while the Teste de Raciocínio Inferencial ranged from zero to 36 . The differences of means were compared between the contrasted groups $(t=-2,47 ; d f=63 ; p=0,017)$, which indicated that one test
\end{abstract}

1 Profesor Del Programa de Estudos Pós-graduados em Psicologia da Universidade São Francisco, campus Itatiba/SP. ; E-maill: fermino.sisto@gmail.com 
could discriminate the other. Besides, a positive and significant coefficient of correlation $(r=0,24 ; p=0,01)$ between total scores of the tests was detected. Therefore, the objective of this study was achieved, since two evidences of validity (a convergent-discriminant and by extreme groups) were found.

Keywords: attention test, validity, psychometric, psychological assessment.

\begin{abstract}
RESUMEN
El objetivo de esta investigación fue buscar evidencias de validez para la Escala de Atenção Seletiva Visual. Primeramente, fue estudiada por grupos de contraste y después correlacionando con el Test de Raciocinio Inferencial. La investigación involucró 119 estudiantes universitarios con promedio de edad de 22,96 ( $\mathrm{DP}=5,67)$, predominantemente del sexo femenino (79\%). Las puntuaciones de la Escala de Atenção Seletiva e Visual variaron entre cuatro y 30 puntos, mientras que las del test de Raciocinio Inferencial se quedaron entre cero y 36 puntos. Fueron comparados los promedios de los grupos de contraste $(t=-2,47$; $\mathrm{gl}=63 ; \mathrm{p}=0,017)$ y el resultado indicó que un test discriminó el otro. Además, fue constatada una correlación positiva y significativa $(r=0,24 ; \mathrm{p}=0,01)$ entre las puntuaciones totales de los dos testes. Por lo tanto, el objetivo de estudio fue logrado, puesto que fue hallada una evidencia de validez convergente-discriminante como también por grupos de contraste.
\end{abstract}

Palabras clave: test de atención, validez, psicometría, evaluación psicológica.

\title{
INTRODUÇÃO
}

Os sentidos humanos recebem, constantemente, uma enorme quantidade de informações que associadas aos estímulos provenientes de processos internos, como a memória, extrapolam a capacidade do organismo de processamento da informação. Diante disso, torna-se necessário efetuar uma seleção desses estímulos distinguindo-os de acordo com sua relevância para uma determinada tarefa ou momento, direcionando os recursos mentais àqueles julgados como relevantes, em detrimento dos avaliados como irrelevantes. Denomina-se atenção, justamente o fenômeno que permite essa seleção e posterior processamento da informação (Sternberg, 2000).

A atenção tem sido considerada uma função neuropsicológica complexa, oriunda de uma predisposição do cérebro para responder determinados estímulos em detrimento de outros (Brandão, 1995). Pode-se defini-la como o fenômeno pelo qual se processa, conscientemente ou não, uma enorme quantidade de informações, internas e externas, disponibilizadas através dos sentidos, memórias e de outros processos cognitivos (Gazzaniga, Ivry \& Mangun, 2006; Sternberg, 2000).

Devido à complexidade do fenômeno atencional várias classificações foram desenvolvidas tendo por base critérios diferentes como seu caráter volitivo ou sua funcionalidade, sendo este, o mais comumente utilizado. Segundo, esse critério encontra-se a atenção dividida, alternada, sustentada e seletiva (Gaddes \& Edgell, 1994; Sternberg, 2000). 
A atenção dividida refere-se à capacidade de selecionar dois ou mais estímulos simultaneamente (Gaddes \& Edgell, 1994; Sternberg, 2000), enquanto na alternada a atenção desloca-se rotativamente de um estímulo a outro (Sternberg, 2000). Define-se atenção sustentada como a possibilidade de manutenção da capacidade atencional durante um longo período (Gaddes \& Edgell, 1994). Por sua vez, entende-se como atenção seletiva a capacidade de selecionar um estímulo dentre vários (Gaddes \& Edgell, 1994; Sternberg, 2000), permitindo checar previsões geradas a partir da memória sobre regularidades passadas do ambiente (Andrade, Santos \& Bueno, 2004).

Dentre os modelos sobre a atenção seletiva visual destacam-se, na atualidade, as pesquisas sobre a atenção espacial em especial aquelas que investigam as propriedades do foco atencional (Moreno \& Marin, 2006). Esses estudos questionam, por exemplo, o tamanho do foco atencional, seu caráter fixo ou flexível, e como ocorre o processamento da informação dentro e fora de seu raio. Inicialmente as propriedades do foco atencional foram explicadas por meio da metáfora do holofote atentivo (attention spotlight model) segundo a qual a atenção poderia ser comparada a um feixe de luz que se desloca sobre uma superfície não iluminada e consegue perceber somente aquilo que é iluminado pelo holofote. O foco atencional teria uma forma circular fixa, de aproximadamente um ângulo de visão. Movendo-se por um campo visual interno de representações, a atenção seleciona e integra os estímulos com base nas suas localizações (Eriksen \& Eriksen, 1974; Rossini \& Galera, 2006; Moreno \& Martin, 2006).

Contudo, posteriormente, surgiram novas evidências indicando que o foco atencional era flexível e sensível às demandas da tarefa, sendo mais bem comparado a uma lente de aumento, pois o foco atencional poderia ser ampliado ou reduzido de acordo com as demandas da tarefa. Além disso, segundo o modelo da lente de aumento (zoom lens model) existiria uma relação inversa entre o tamanho do foco atentivo e a intensidade do processamento da informação; com um foco atentivo reduzido a informação seria fortemente processada, e com um foco atentivo ampliado menos recursos seriam empregados em cada uma das localizações ou estímulos da área abarcada (Castiello \& Umiltà, 1990; Eriksen \& St. James, 1986; Eriksen \& Yeh, 1985; Shulman \& Wilson, 1987).

Hilgard e Atkinson (1979) apontaram que as operações intelectuais, tal como a inteligência, também estão envolvidos no processamento da atenção. Existem diversas teorias sobre a inteligência, no entanto, não se pode deixar de citar a "Teoria Bifatorial" ou "Teoria Eclética dos Fatores" de Charles Spearman, de 1904.

$\mathrm{Na}$ Teoria Bifatorial Spearman diferenciou três fatores, a saber, o fator "g", comum a todos as habilidades do homem, o fator "e", ou o fator especifico limitado para cada habilidade e, por último, os fatores de grupos, que seriam determinados por um grande número de fatores. O instrumento de inteligência utilizado neste estudo apresentou características que podem ser consideradas como saturado no fator "g" (Sisto, 2006). 
Diversos autores procuraram uma relação entre a inteligência e a atenção, como König, Bühner e Mürling (2005) que estudaram qual o melhor preditor para o desempenho em tarefas múltiplas, sendo elas a atenção, a memória de trabalho e a inteligência fluida. $\mathrm{O}$ estudo foi realizado com 122 sujeitos e foi aplicado o Simultaneous capacity/Multitasking. A análise de regressão hierárquica múltipla mostrou que o melhor preditor foi a memória de trabalho com adição de atenção e inteligência.

Em um estudo com 435 crianças, Pascualvaca e cols. (1997) aplicaram uma bateria de atenção para investigar a influência de sexo e inteligência verbal na atenção. Os resultados mostraram que a atenção recebe a influência do sexo e da inteligência verbal, sendo que criança com altas pontuações no teste de inteligência verbal foram melhores no teste de atenção. Todavia, Weyandt, Mitzlaff e Thomas (2002) estudaram em 79 pessoas, sendo 17 com déficit de atenção e hiperatividade, a relação entre inteligência e atenção em jovens adultos. Foi utilizado o Test of Variables of Attention. Os resultados indicaram que não houve nenhuma correlação significativa entre inteligência e a atenção.

Sisto, Rueda, Noronha e Bartholomeu (2007) estudaram as relações entre a inteligência e a atenção dividida. Foram aplicados em 215 sujeitos o Teste de Atenção Dividida (AD) e o Teste Conciso de Raciocínio (TCR). Os resultados mostraram correlações positivas entre o TCR e o $\mathrm{AD}$. Em relação à medida de Concentração, o coeficiente de correlação encontrado foi de $0,47(p=0,000)$, e na Velocidade com Qualidade de 0,34 $(\mathrm{p}=0,000)$.

Em outro estudo, Rueda, Lamounier, Sisto, Bartholomeu e Noronha (2006) verificaram a relação entre inteligência e atenção sustentada. Foram aplicados o Teste de Atenção Sustentada (AS) e o Teste Conciso de Raciocínio em 217 sujeitos. Foram encontradas correlações positivas significativa entre o TCR e as medidas atencionais fornecidas pelo AS, sendo o coeficiente de correlação de $0,42(p=0,000)$ em relação a Concentração, e de $0,40(p=0,000)$ na Velocidade com Qualidade.

Silva (2008) correlacionou o Teste R1, que avalia o fator g, com o Teste de Atenção Concentrada (AC), e o Teste de atenção (TADIM) em uma amostra de motoristas. Participaram do estudo 68 motoristas, do sexo masculino, com idade média de 21,72 anos, todos residentes no estado do Rio Grande do Norte. A avaliação ocorreu em dois momentos, durante o processo de concessão da carteira de motorista, e após cinco anos. Foram encontradas correlações nulas e baixas entre o R1 e o AC $(>-0,15<0,39)$ e entre nula e moderada com o TADIM $(>-0,17<0,68)$.

Ainda, Sisto (2006) verificou a relação entre e o Teste Conciso de Raciocínio e o Teste de Atenção Concentrada (AC) em 118 participantes, sendo que os resultados indicaram uma correlação positiva de $0,45(\mathrm{p}=0,000)$ entre os testes. Nessa linha, Cecilio-Fernandes e Rueda (2007) estudaram em 78 universitários a relação entre o Teste de Atenção Concentrada - TEACO-FF e o Teste Conciso de Raciocínio TCR. Os resultados apontaram uma correlação positiva e significativa, de baixa magnitude. 
Estudos que adotaram outros paradigmas sobre a inteligência também encontraram associações entre inteligência e a capacidade atencional. Weyandt, Mitzlaff e Thomas (2002), por exemplo, associaram a Wechler Adult Intelligence ScaleRevised (WAIS-R) ao Test of Variables of Attention (TOVA). Participaram desse estudo 17 pessoas com TDAH e 62 universitários entre 18 e $52(\mathrm{M}=25,5)$ anos. Quando comparados os grupos nas medidas de atenção oferecidas pelo TOVA e de inteligência os resultados indicaram diferenças significativas apenas para a omissão de resposta, com vantagem para o grupo sem TDAH. Ainda, as correlações entre os dois testes foram nulas, com índices até 0,15 .

Savage, Cornish, Manly e Hollis (2006) utilizaram a Wechsler Abbreviated Scale Intelligence (WASI) para investigar a relação entre inteligência, atenção dividida e controle inibitório, memória de trabalho e aquisição de leitura em 123 crianças com e sem problemas de atenção. Eles identificaram que a memória de trabalho e a atenção foram os melhores indicadores de para a diferenciação entre o grupo controle e os participantes com TDAH, sendo que, as crianças com dificuldades de atenção obtiveram piores desempenhos no WAIS. No que concerne à correlação entre atenção e inteligência, os índices foram estatisticamente significativos com magnitudes variando entre 0,39 e 0,62 .

Tonelotto (2001), por sua vez, comparou o desempenho na Escala Wechsler de Inteligência para Crianças (WISC) de dois grupos de crianças com e sem indicativo de problemas de atenção. O primeiro grupo foi formado com 10 participantes, e o segundo com 19. Das três dimensões propostas pelo WISC foram observadas diferenças significativas em duas, QI Verbal e Total, com melhor desempenho para o grupo sem indícios de problema de atenção.

De modo geral, as pesquisas que associaram inteligência e atenção encontraram resultados indicativos de uma correlação positiva entre eles, independentemente do tipo de atenção avaliado ou da perspectiva adotada sobre a inteligência. Entretanto, a magnitude dessa correlação apresentou-se bastante diversa, o que se justifica, apesar da aparente contradição, pelas diferenças individuais e complexidade da tarefa em ambos os processos. Segundo Rosselló e Munar (1994), embora a relação entre atenção e inteligência seja verificada mesmo em tarefas mais simples, a complexidade da tarefa atencional interferiria na associação entre elas. Nesse sentido, essa relação acentuar-se-ia em tarefas que exigissem rapidez, como as avaliadas por tempo de reação. Ainda, tal acentuação ocorreria em amostras com maior variação nos níveis inteligência.

Dentro desse contexto, esta pesquisa teve como objetivo investigar a existência de relação entre a atenção e a inteligência, procurando evidência de validade para a Escala de Atenção Seletiva Visual (EASE). Para isso, o teste de Raciocínio Inferencial foi escolhido como critério, uma vez que o mesmo já apresenta estudos com testes que avaliam a atenção. Acrescente a isso, a análise de grupos extremos, a qual forneceria outra evidência de validade. 


\section{MÉTODO}

\section{Participantes}

Participaram da pesquisa 119 universitários de uma universidade particular do interior de São Paulo. A média de idade foi de 22,96 (DP=5,67), mínima de 18 e máxima de 56 anos, sendo $94(79,00 \%)$ do sexo feminino. Houve uma concentração entre os participantes mais jovens, pois, $94(79,00 \%)$ ficaram entre 18 e 25 anos.

\section{Instrumentos}

Escala de Atenção Seletiva Visual - EASE (Castro, 2008).

Para a construção do EASE foi adotado o modelo da lente de aumento, pretendendo interferir no tamanho do foco atentivo controlando as dimensões do estímulo por meio de sua quantidade em cada item (Castro, 2008). Para a construção dos itens foram controladas duas variáveis, número de estímulos distratores, por meio do qual se pretendeu promover modificações no foco atencional, e número de estímulos-alvo. Para a variável número de estímulos distratores ocorreu a possibilidade de três, seis ou nove estímulos, e para o número de estímulos-alvo um, dois ou três. Para cada item apresentou-se um estimulo modelo e quatro opções de resposta. A tarefa consistiu em identificar a opção de resposta com mais estímulos coincidentes com o modelo. Desse modo, ao serem combinadas as duas variáveis houve nove tipos de itens, sendo, 333, 332, 331, 663, 662, 661, 993, 992 e 991. $\mathrm{O}$ primeiro número à esquerda corresponde ao número de estímulos distratores presentes na figura modelo, o segundo ao número de estímulos distratores nas opções de resposta, e o número à direta representa a quantidade de estímulos-alvo a serem localizados. Com base em estudos preliminares os itens do tipo 333, $661 \mathrm{e}$ 991 foram excluídos por terem sido avaliados como pouco discriminativos. Dessa maneira, o instrumento constitui-se por seis tipos de itens: 332, 331, 663, 662, 993 e 992 . Foram oferecidos cinco itens para cada tipo, em um total de 30 itens.

Estudo realizado com 120 universitários de diversos cursos investigou a relação entre o número de estímulos distratores e número de estímulos-alvo com o nível de dificuldade dos itens atribuído segundo o Modelo Rasch, com vistas a obter evidência de validade pelo processo de resposta. Os resultados indicaram uma relação direta entre o nível de dificuldade dos itens e as variáveis número de estímulos distratores $[\mathrm{F}(2,119)=3327,27 ; \mathrm{p}<0,000]$ e número de estímulosalvo $[\mathrm{F}(2,119)=1524,69 ; \mathrm{p}<0,000]$ diferenciando-se todos os grupos. Além disso, cada um dos seis tipos de itens se diferenciou $[\mathrm{F}(5,119)=2422,75 ; \mathrm{p}<$ $0,000]$ apresentando níveis de dificuldade diferentes conforme seqüência a seguir em ordem crescente: 332, 331, 662, 663, 993 e 992.

\section{Teste de Raciocínio Inferencial - Rin (Sisto, 2006)}

Consiste em um teste de 40 itens, sendo cada item uma seqüência de figuras geométricas que formam uma seqüência lógica. O candidato tem que completá-las escolhendo uma das alternativas. Ainda, o instrumento é dividido em quatro partes, sendo que a primeira parte é mais fácil e a ultima parte é a mais difícil. A aplicação 
tem tempo máximo de 25 minutos. No inicio são apresentadas as instruções e em seguida há um exemplo para verificar a compreensão das instruções. Na correção é atribuído um ponto para cada acerto, e no final somam-se todos os acertos. No que tange os parâmetros psicrométricos, o instrumento possui estudos validade, precisão e de padronização.

\section{Procedimento}

A coleta de dados realizou-se coletivamente em sala de aula e foi precedida pela obtenção do Termo de Consentimento Livre e Esclarecido. Aplicou-se, primeiramente, o RIn, com duração de 25 minutos e em seguida o EASE com tempo livre.

\section{RESULTADOS}

A análise dos dados iniciou-se pela descrição dos resultados nos dois testes. $\mathrm{Na}$ Tabela 1 encontram-se as estatísticas descritivas do EASE por tipo de item, por número de estímulos distratores e de estímulos-alvo e por total e, para o RIn, essas informações foram oferecidas por série e total.

Observa-se que o EASE apresentou médias bastante elevadas em todas as suas medidas secundárias (tipos de itens, número de estímulos distratores e estímulosalvo), na maioria dos casos com valores médios muito próximos à pontuação total da categoria. Como exemplo pode-se citar os tipos de itens. Nesse caso, em cada tipo os participantes poderiam fazer cinco pontos, sendo que as médias ficaram em torno de quatro pontos, em sua maioria, ou bem próximas de cinco (tipos 32 e 33). Mas, vale destacar, que se observou uma tendência a médias menores para as categorias mais difíceis.

Esse quadro não se repetiu com as séries do RIn. Elas apresentaram médias com valores intermediários em relação à pontuação total possível, com uma tendência a médias proporcionalmente menores para as séries mais difíceis.

Tabela 1. Estatística descritiva do EASE e RIn.

\begin{tabular}{|c|c|c|c|c|c|c|c|}
\hline \multicolumn{8}{|c|}{ EASE } \\
\hline & \multicolumn{6}{|c|}{ Tipos de itens } & \multirow{2}{*}{ Total } \\
\hline & 32 & 31 & 63 & 62 & 93 & 92 & \\
\hline Média & 4,82 & 4,53 & 3,87 & 4,08 & 3,68 & 3,60 & 24,57 \\
\hline Desvio padrão & 0,62 & 1,15 & 1,37 & 1,24 & 1,51 & 1,55 & 5,92 \\
\hline Erro padrão médio & 0,057 & 0,105 & 0,126 & 0,113 & 0,139 & 0,142 & 0,542 \\
\hline Mínimo & 0 & 0 & 0 & 0 & 0 & 0 & 4 \\
\hline \multirow[t]{3}{*}{ Máximo } & 5 & 5 & 5 & 5 & 5 & 5 & 30 \\
\hline & \multicolumn{3}{|c|}{$\mathrm{N}^{0}$ de estímulos distratores } & \multicolumn{3}{|c|}{$\mathrm{N}^{\circ}$ de estímulos-alvo } & \\
\hline & 9 & 6 & 3 & 1 & 2 & 3 & \\
\hline Média & 7,28 & 7,94 & 9,35 & 13,59 & 12,50 & 11,32 & \\
\hline Desvio padrão & 2,81 & 2,36 & 1,58 & 3,45 & 2,83 & 3,78 & \\
\hline
\end{tabular}




\begin{tabular}{|c|c|c|c|c|c|c|}
\hline Erro padrão médio & 0,257 & 0,216 & 0,144 & 0,316 & 0,260 & 0,347 \\
\hline Mínimo & 1 & 0 & 2 & 0 & 2 & 0 \\
\hline Máximo & 10 & 10 & 10 & 15 & 15 & 15 \\
\hline \multicolumn{7}{|c|}{ RIn } \\
\hline & Série A & Série B & Série C & Série D & Total & \\
\hline Média & 8,27 & 4,71 & 2,77 & 5,85 & 60 & \\
\hline Desvio padrão & 2,50 & 1,97 & 1,41 & 2,82 & 6,30 & \\
\hline Erro padrão médio & 0,229 & 0,180 & 0,129 & 0,258 & 0,578 & \\
\hline Mínimo & 0 & 0 & 0 & 0 & 0 & \\
\hline Máximo & 12 & 8 & 5 & 12 & 36 & \\
\hline
\end{tabular}

Em se tratando das pontuações totais, o EASE apresentou um resultado bastante elevado (Figura 1). As pontuações variaram de 4 a 30, com média de 24,57 pontos $(\mathrm{DP}=5,92)$, sendo que $65(54,60 \%)$ participantes ficaram entre 26 e 30 pontos. Como o escore máximo do EASE é de 30 pontos, esses resultados indicaram uma tendência ao efeito teto. Essas informações podem ser mais bem visualizadas na Figura 1.

A distribuição dos participantes pela pontuação total do RIn foi mais homogênea, variando de zero à 36 pontos, com média de $21,60(\mathrm{DP}=6,30)$. Dos participantes, $48(40,30 \%)$ ficaram entre 21 e 25 pontos, sendo que $31(33,60 \%)$ ficaram abaixo de 21 pontos e $40(26,10 \%)$ acima de 25 .

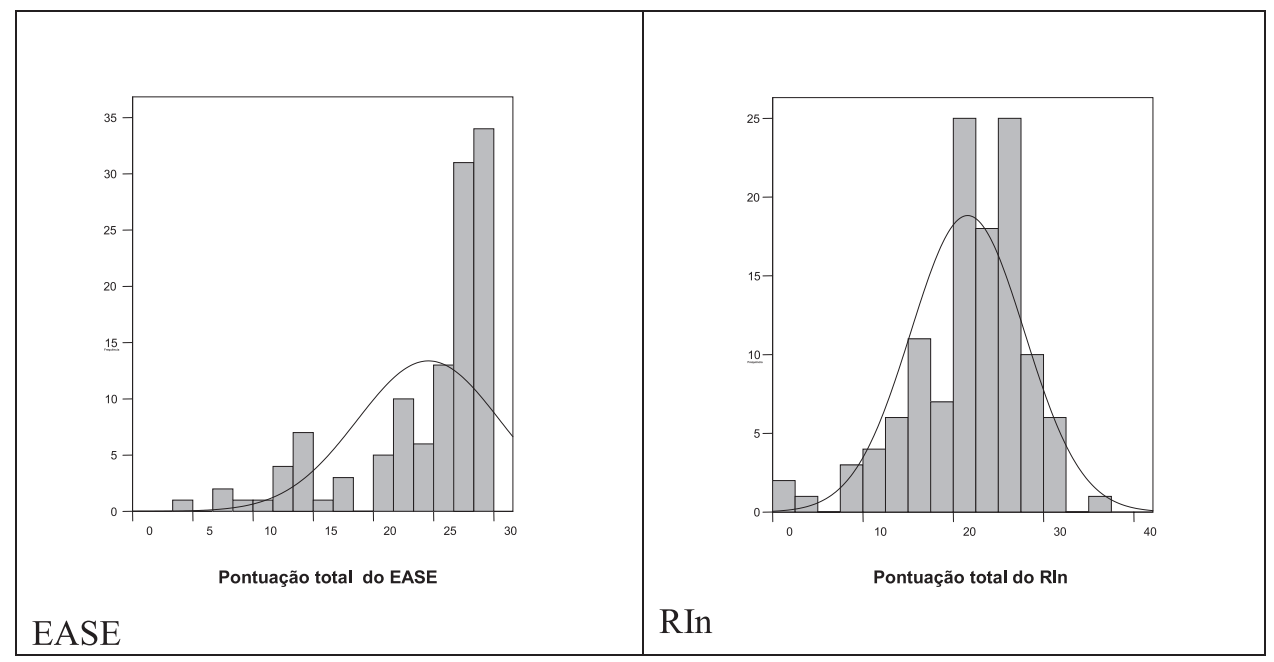

Figura 1. Histograma com a pontuação total do EASE e RIn.

Realizou-se um teste t de Student para verificar se grupos extremos no RIn se diferenciariam em razão de suas pontuações totais no EASE. O Grupo 1 foi composto por pessoas que obtiveram escores entre 0 e 19 e o Grupo 2 por pessoas que obtivera escores entre 26 e 36 . Houve diferença significativa $(t=-2,47 ; \mathrm{gl}=63$; 
$\mathrm{p}=0,017)$, sendo que o $\mathrm{G} 1(\mathrm{M}=22,35, \mathrm{DP}=6,70)$ teve média menor que o $\mathrm{G} 2(\mathrm{M}=26,06 ; \mathrm{DP}=5,34)$, ou seja, o grupo com menores pontuações no RIn obteve menor média no EASE.

Foram calculados coeficientes de correlação de Pearson entre as pontuações do EASE e do RIn. Além da pontuação total, o EASE permitiu análises estatísticas com os itens agrupados por número de estímulos distratores (33, 66 e 99), número de estímulos-alvo (1, 2 e 3 ) e tipo de itens $(332,331,663,662,993$ e 992). Por sua vez, do RIn também foram usadas medidas com base em cada série e em relação ao total. As correlações entre o Teste de Atenção seletiva Visual e o RIn foram positivas e, em sua maioria, significativas (20 em 60 coeficientes) e de magnitude baixa ou moderada (Tabela 2).

Tabela 2. Correlações de Pearson entre o EASE e RIn.

\begin{tabular}{|c|c|c|c|c|c|c|c|}
\hline \multirow{2}{*}{ EASE } & & & \multicolumn{5}{|c|}{ RIn } \\
\hline & & & Série A & Série B & Série C & Série D & RIn total \\
\hline \multirow{6}{*}{$\begin{array}{l}\text { Estímulos } \\
\text { distratores }\end{array}$} & \multirow{2}{*}{33} & $\mathrm{R}$ & 0,21 & 0,28 & 0,24 & 0,07 & 0,15 \\
\hline & & $\mathrm{P}$ & 0,023 & 0,002 & 0,009 & 0,466 & 0,097 \\
\hline & \multirow{2}{*}{66} & $\mathrm{R}$ & 0,26 & 0,25 & 0,22 & 0,14 & 0,20 \\
\hline & & $\mathrm{P}$ & 0,005 & 0,007 & 0,017 & 0,124 & 0,027 \\
\hline & \multirow{2}{*}{99} & $\mathrm{R}$ & 0,29 & 0,27 & 0,36 & 0,07 & 0,24 \\
\hline & & $\mathrm{P}$ & 0,002 & 0,003 & 0,000 & 0,451 & 0,009 \\
\hline \multirow{6}{*}{ Estímulos-alvo } & \multirow{2}{*}{1} & $\mathrm{R}$ & 0,26 & 0,26 & 0,28 & 0,07 & 0,16 \\
\hline & & $\mathrm{P}$ & 0,005 & 0,004 & 0,002 & 0,436 & 0,092 \\
\hline & \multirow{2}{*}{2} & $\mathrm{R}$ & 0,29 & 0,25 & 0,31 & 0,12 & 0,24 \\
\hline & & $\mathrm{P}$ & 0,001 & 0,005 & 0,001 & 0,189 & 0,010 \\
\hline & \multirow{2}{*}{3} & $\mathrm{R}$ & 0,24 & 0,30 & 0,27 & 0,08 & 0,22 \\
\hline & & $\mathrm{p}$ & 0,008 & 0,001 & 0,003 & 0,367 & 0,018 \\
\hline \multirow{12}{*}{ Tipo de itens } & \multirow{2}{*}{32} & $\mathrm{r}$ & 0,05 & 0,24 & 0,09 & 0,04 & 0,10 \\
\hline & & $\mathrm{p}$ & 0,569 & 0,010 & 0,331 & 0,681 & 0,275 \\
\hline & \multirow{2}{*}{31} & $\mathrm{r}$ & 0,26 & 0,26 & 0,28 & 0,07 & 0,16 \\
\hline & & $\mathrm{p}$ & 0,005 & 0,004 & 0,002 & 0,436 & 0,092 \\
\hline & \multirow{2}{*}{63} & $\mathrm{r}$ & 0,23 & 0,29 & 0,21 & 0,14 & 0,21 \\
\hline & & $\mathrm{p}$ & 0,013 & 0,001 & 0,020 & 0,124 & 0,020 \\
\hline & \multirow{2}{*}{62} & $\mathrm{r}$ & 0,24 & 0,15 & 0,18 & 0,11 & 0,15 \\
\hline & & $\mathrm{p}$ & 0,009 & 0,115 & 0,049 & 0,222 & 0,104 \\
\hline & \multirow{2}{*}{93} & $\mathrm{r}$ & 0,20 & 0,24 & 0,26 & 0,01 & 0,17 \\
\hline & & $\mathrm{p}$ & 0,029 & 0,008 & 0,004 & 0,910 & 0,070 \\
\hline & \multirow{2}{*}{92} & $\mathrm{r}$ & 0,32 & 0,25 & 0,39 & 0,12 & 0,27 \\
\hline & & $\mathrm{p}$ & 0,000 & 0,005 & 0,000 & 0,210 & 0,003 \\
\hline \multirow{2}{*}{ Total } & & $\mathrm{r}$ & 0,29 & 0,30 & 0,32 & 0,11 & 0,24 \\
\hline & & $\mathrm{p}$ & 0,001 & 0,001 & 0,000 & 0,244 & 0,010 \\
\hline
\end{tabular}


As exceções ficaram para as correlações entre a Série A e o tipo 332, a Série B e o tipo 662, a Série C e os tipos 332 e 662, a pontuação total do RIn e o grupo de estímulos distratores 33, o grupo com 1 estímulo-alvo e os tipos 332, 331, 662 e 993, além de todas as correlações com a Série D que foram nulas. Destaca-se que a correlação entre a pontuação total do EASE e do RIn e suas séries variaram de 0,24 à 0,32 , excetuando a Série D.

\section{DISCUSSÃO}

A relação entre os construtos de atenção e inteligência foi mostrado, na literatura, por diversos pesquisadores como, por exemplo, König, Bühner e Mürling (2005), Pascualvaca e cols. (1997), Tonelotto (2001). Este estudo teve por objetivo, justamente, relacionar a inteligência avaliada pelo teste de Raciocínio Inferencial (Sisto, 2006) com a atenção seletiva, mensurada pela Escala de Atenção Seletiva Visual (Castro, 2008).

Quando comparados os instrumentos foi verificada uma correlação positiva e significativa entre a pontuação total de ambos os testes. Nesse sentido, os resultados vão ao encontro dos achados de Rueda, Lamounier, Sisto, Bartholomeu e Noronha (2006), Sisto (2006), Sisto, Rueda, Noronha e Bartholomeu (2007) e Cecilio-Fernandes e Rueda (2007).

Observou-se que as correlações dos conjuntos de itens do EASE segundo os critérios número de estímulos distratores, de estímulos-alvo e tipo de item com as séries $\mathrm{A}, \mathrm{B}$ e $\mathrm{C}$ e a pontuação total do RIn encontraram índices de magnitude baixa, em sua maioria, e alguns de magnitude nula, o que ocorreu com os conjuntos de itens mais fáceis do EASE. Além disso, todas as correlações com a série D foram nulas. Duas informações podem auxiliar na compreensão desses resultados. A primeira refere-se ao fato de que as pontuações no EASE foram bastante elevadas, possivelmente pela forma de administração do teste que adotou o tempo livre. A segunda refere-se à própria estrutura dos dois testes.

Sobre isso se deve considerar que os itens do EASE foram construídos tendo por base o modelo da lente de aumento (Castro, 2008), segundo o qual o tamanho do foco atencional adapta-se às demandas da tarefa (Castiello \& Umiltà, 1990; Eriksen \& St. James, 1986; Eriksen \& Yeh, 1985; Shulman \& Wilson, 1987). Desse modo, os conjuntos de itens do EASE apresentam níveis de dificuldade diferentes em razão do número de estímulos distratores, de estímulos-alvo e pelo tipo de item formado pela combinação dessas variáveis (Castro, 2008). Também os itens do RIn diferenciam-se em razão do seu nível de dificuldade, nesse caso organizados em quatro séries, sendo a série D a mais difícil (Sisto, 2006).

Somando-se essas informações pode-se hipotetizar que o EASE, conforme administrado neste estudo, elevou o nível de acertos superestimando a capacidade atencional dos participantes não os discriminando adequadamente. Isso gerou menor variabilidade intra-grupos e, desse modo, interferiu nos índices de correlação. Assim, um novo estudo entre o EASE, dessa vez com tempo de execução 
delimitado, e o RIn deverá verificar se uma melhor distribuição dos participantes promoverá mudanças nessas correlações.

Ainda, os resultados indicaram que grupos com pontuações extremas no RIn, ou seja, pontuações muito baixas e muito elevadas, obtiveram desempenhos estatisticamente distintos no EASE, indicando que pessoas com baixo rendimento no RIn também o terá no EASE, sendo o inverso verdadeiro. Esse resultado concordaram com outros estudos que identificaram piores desempenhos em tarefas que avaliavam inteligência, para pessoas com dificuldades de atenção (Tonelotto, 2001; Savage, Cornish, Manly, \& Hollis, 2006).

Com isso exposto, dois pontos podem ser destacados. O primeiro, que os achados neste estudo corroboram a literatura na tendência de confirmar uma relação entre os construtos, mesmo sendo formas diferentes de atenção. Outro ponto a destacar, seria a correlação entre ambos os testes, apontado para uma evidência de validade convergente-divergente para o EASE. Ainda, observou-se outra evidência de validade, por grupos extremos. Por fim, o objetivo do estudo foi alcançado, e sugere-se novas pesquisas para o teste de atenção seletiva, para analises mais aprofundadas.

\section{REFERÊNCIAS}

Andrade, V. M., Santos, F. H., \& Bueno, O. F. A. (2004). Neuropsicologia Hoje. São Paulo: Artes Médicas.

Brandão, M. L. (1995). Atenção. In: Brandão, M. L. (org.) Psicofisiologia. (pp. 145154). São Paulo: Atheneu.

Castiello, U., \& Umiltà, C. (1990). Size of the attentional focus and efficiency of processing. Acta Psychologica, 73, 195-209.

Castro, N. R. (2008). Construção de um teste de atenção seletiva visual. Dissertação de mestrado, Universidade São Francisco, Brasil.

Cecilio-Fernandes, D., \& Rueda, F. J. M. (2007). Evidência de validade concorrente para o Teste de Atenção Concentrada - TEACO-FF. Revista Psic, 8(2), 167-174.

Eriksen, B., \& Eriksen, C. (1974). Effects of noise letters upon the identification of a target letter in a nonsearch task. Perception \& Psychophysics, 16(1), 143-149.

Eriksen, C., \& St. James, J. (1986). Visual attention within and around the field of focal attention: a zoom lens model. Perception \& Psychophysics, 40(4), 225-240.

Eriksen, C., \& Yeh, Y. Y. (1985). Allocation of attention in the visual field. Journal of Experimental Psychology: Human Perception and Performance, 11(5), 583-597.

Gazzaniga, M. S., Ivry, R. B., \& Mangun, G. R. (2006). Neurociência Cognitiva: a Biologia da mente. Tradução Angélica Rosat Consiglio $2^{\mathrm{a}}$ edição. Porto Alegre: Artmed.

Hilgard, E. R. \& Atkinson, R. C. (1979). Introdução à psicologia. São Paulo: Editora Nacional. 
KÖNIG, C. J., BÜHNER, M. \& MÜRLING, G. (2005). Working Memory, Fluid Intelligence, and Attention Are Predictors of Multitasking Performance, but Polychronicity and Extraversion Are not. Human Performance, 18(3), 243-266

Moreno, A. C., \& Marin, A. P. (2006). Redes atencionales y sistema visual selectivo. Revista da Univ. Psychol. Bogotá, 5(2), 305-325.

Pascualvaca, D. M., Anthony, B. J., Arnold, L. E., Rebok, G. W., Ahearn, M. B., Kellan, S. P. \& Mirsky, A. F. (1997). Attention performance in an epidemiological sample of urban children: the role of gender and verbal intelligence. Child Neuropsychology, 3(1), 13-27.

Posner, M. I. (1992). Attention as a Cognitive and Neural System. Current Directions in Psychological Science, 1(1), 11-14.

Rosselló, J, \& Roca, M. (1994). El mecanismo atencional: estudo de lãs diferencias individuales. Revista de Psicología General y Aplicada, 47(4), 383-390.

Rossini, J. C., \& Galera, C. (2006). Atenção visual: estudos comportamentais da seleção baseada no espaço e no objeto. Estudos de Psicologia (Natal), 11(1), 79-86.

Rueda, F. J. M., Lamounier, R., Sisto, F. F., Bartholomeu, D. \& Noronha, A. P. P. (2006). Raciocínio inferencial e atenção sustentada: construtos convergentes? Em C. Machado, L. Almeida, M. A. Guisande, M. Gonçalves \& V. Ramalho. (Org.). XI Conferência Internacional. Avaliação Psicológica: formas e contextos. Braga: Psiquilibrios.

Savage, R., Cornish, K., Manly, T., \& Hollis, C. (2006). Cognitive processes in children's reading and attention: the role of working memory, divided attention, and response inhibition. British Journal of Psychology, 97, 365-385.

Shulman, G. L., \& Wilson, J. (1987). Spatial frequency and selective attention to spatial location. Perception, 1(16), 103-111.

Silva, F. H. V. C. (2008). Medidas da preditividade de instrumentos psicológicos nas avaliações psicológicas de condutores. Dissertação de Mestrado, Universidade Federal do Rio Grande do Norte.

Sisto, F. F., (2006). Teste Conciso de Raciocínio. Manual. São Paulo: Vetor Editora Psicopedagógica Ltda.

Sisto, F. F., Rueda, F. J. M., Noronha, A. P. P. \& Bartholomeu, D. (2007). Atenção Dividida e Inteligência: evidências de validade. Encontro (Santo André), XI(16), 117-131.

Sternberg, R. J. (2000). Psicologia Cognitiva. Porto Alegre: Artmed Editoras.

Tonelotto, J. M. F.(2001). A utilidade do WISC na detecção de problemas de atenção em escolares. Psicologia escolar educacional, 5(2), 31-37.

Weyandt, L. L., Mitzlaff, L., \& Thomas, L. (2002). The relationship between intelligence and performance on the Test of Variables of Attention (TOVA). Journal of learning Disabilities, 35(2), 114-120. 